\title{
Anesthetic Management of a Patient With Spasmodic Torticollis Treated With a Deep Brain Stimulator Implant Operated for Inguinal Hernia
}

\author{
Athanasia Tsaroucha ${ }^{\mathrm{a}, \mathrm{b}}$, Titika Moschovou ${ }^{\mathrm{a}}$, Aikaterini Melemeni ${ }^{\mathrm{a}}$, \\ Eriphili Argyra ${ }^{\mathrm{a}}$
}

\begin{abstract}
Spasmodic torticollis (ST) is the most frequent and familiar type of focal dystonia. Nowadays, deep brain stimulation (DBS) seems to be an effective treatment for ST and is considered a first-line therapy. Anesthesia in such patients requires special considerations, because of the characteristics of the disease as well as the respective treatment. We present the anesthetic management of a patient with ST treated with DBS. A 53-year-old man with idiopathic cervical dystonia treated with a deep brain stimulator device underwent an inguinal hernia correction under general anesthesia. A detailed preanesthesia evaluation was performed to assess any issues associated with the disease especially concerning the airway. A total intravenous anesthesia technique was planned. The device was turned off immediately after induction and turned on before emergency. The patient did not report any discomfort and the stimulator did not present any malfunction or interference with other devices. Anesthetic management of patients with ST treated with deep brain stimulator should focus on a careful preanesthesia evaluation regarding primarily the airway examination, a detailed planning of the anesthetic technique to be applied, and a correct handling of the DBS implanted as it can interfere with other monitoring and therapeutic devices, sometimes with severe consequences.
\end{abstract}

Keywords: Spasmodic torticollis; Deep brain stimulation; Anesthesia; Inguinal hernia

\section{Introduction}

Dystonias are a wide group of movement disorders in which persistent involuntary muscle contractions lead to abnormal postures and repetitive movements [1]. Classification schemes

\footnotetext{
Manuscript accepted for publication July 13, 2016

aDepartment of Anesthesiology, Aretaieio Hospital, Medical School, National and Kapodistrian University of Athens, Greece

bCorresponding Author: Athanasia Tsaroucha, Department of Anesthesiology, Aretaieio Hospital, Medical School, National and Kapodistrian University of Athens, Greece. Email: ntsaroucha@yahoo.com
}

doi: http://dx.doi.org/10.14740/jmc2588w are relative to the age of onset ("early" or "late"), the anatomic distribution ("generalized" or "focal") and the etiology ("primary" or "secondary") [2]. Cervical dystonia, also known as "spasmodic torticollis (ST)", is the most common primary focal dystonia [1].

Deep brain stimulation (DBS) uses an implanted medical device to deliver precisely targeted electrical stimulation to the brain to treat the symptoms of movement disorders [3]. Even if it has been mostly applicated for the treatment of tremors and Parkinson's disease, currently it is considered an alternative and effective treatment in medication-resistant forms of dystonia [4].

Since DBS procedures are currently increasing, anesthesiologists must be aware of both the distinguishing characteristics of these "brain pacemakers" and of the patients carrying them. As there is little information and no standard anesthetic guidelines available on patients with a DBS implant who are present for surgery, a careful management is essential to avoid complications [5].

\section{Case Report}

The patient gave written informed consent for this report.

A 53-year-old man suffering from ST with implanted DBS electrode was scheduled for right inguinal hernia. He had been suffering from left-sided ST for the past 7 years in the context of idiopathic focal dystonia. His symptoms were not adequately controlled with pharmacological treatment and consequently he underwent DBS implantation surgery 2 years later, after which his dystonia was noticeably improved. The pulse generator had been placed in the thoracic wall but the patient was unable to indicate where the leads were implanted.

The preoperative neurological visit confirmed the leftsided ST along with a well tolerable muscle rigidity of both upper and lower extremities with hypertonic deep tendon reflexes and clonus and an ataxia syndrome of cerebellar type. The DBS device was controlled and found well functioning.

A careful and detailed preanesthesia evaluation was performed to assess any issues associated with the ST, concerning especially the airway. The airway assessment showed a Mallampati score II, a slightly reduced thyromental distance of 5.5 $\mathrm{cm}$ and limitation in the maximal mouth opening (2.5 patient's fingers). The patient was also unable to extend his neck due to 
the sustained abnormal head posture.

He was under treatment for arterial hypertension for the past 4 years with a combination of an angiotensin converting enzyme inhibitor and a b blocker. ECG revealed sinus rhythm at 75 beats per minute.

His chest X-ray showed the device with leads in the left upper chest. Laboratory results were normal.

During the preoperative visit, the anesthesiologist informed the patient that an awake fiberoptic intubation would be necessary, due to the presence of risk factors for difficult mask ventilation and intubation. He refused to be intubated awake despite the adequate counseling. As he has been intubated before for the DBS implantation, we decided to proceed with routine induction, but with a C-MAC video laryngoscope immediately available.

On the arrival of the patient in the operating room, basic monitoring was applied (ECG, non-invasive blood pressure, pulse oximeter $\mathrm{SpO}_{2}$, end tidal $\mathrm{CO}_{2}$, and bispectral index monitoring BIS). An 18-G peripheral vein catheter was installed and a peripheral nerve stimulator was attached to the right ulnar nerve.

A total intravenous anesthesia technique (TIVA) was planned. Right before induction, the patient received midazolam $1 \mathrm{mg}$ and fentanyl $100 \mu \mathrm{g}$, while anesthesia was induced with propofol $2.5 \mathrm{mg} / \mathrm{kg}$. The DBS device was switched off by a specialized technician 1 min after induction and not before, to avoid any distress from the resulting exacerbation of dystonia. The tracheal intubation was facilitated with rocuronium bromide $70 \mathrm{mg}$ and an $8.5 \mathrm{~mm}$ cuffed tracheal tube was placed uneventfully. Anesthesia was maintained with continuous propofol infusion and titrated at a rate between 30 and 40 $\mathrm{mL} / \mathrm{h}$ to maintain BIS values of between 40 and 60 . An air in oxygen mixture was delivered at a $1 / 1$ ratio.

A bipolar cautery was used to avoid any interference with the DBS device. Additionally, the patient received $10 \mathrm{mg}$ rocuronium bromide, $150 \mu \mathrm{g}$ fentanyl, 1,000 $\mathrm{mg}$ paracetamol and $40 \mathrm{mg}$ parecoxib sodium. He remained hemodynamically stable throughout the whole procedure. At the end of the operation that lasted $1 \mathrm{~h}$ and $20 \mathrm{~min}$, the propofol infusion was stopped. When the first twitch of the train-of-four stimulation appeared, the DBS device was switched on and the neuromuscular block was antagonized with sugammadex $140 \mathrm{mg}$. Dystonia was present for a few seconds right before and right after the activation of the device. The patient emerged smoothly and was extubated 20 min later uneventfully. He did not present any respiratory difficulties and was then shifted in our post-anesthesia care unit. His DBS device did not present any malfunction and he was discharged the next morning after an uneventful recovery.

\section{Discussion}

ST, also called cervical dystonia, distinguishly manifests as a state of sustained and involuntary contractions of the sternocleidomastoid, trapezius, and scalenus muscles resulting in twisting movements of the neck and abnormal posture of the head [1].

Classification of dystonias is based on the age of onset ("early" or "late"), the anatomic distribution ("generalized" or "focal") and the etiology ("primary" or "secondary") [2].

Cervical dystonia is the most common primary focal dystonia [1]. Our patient was affected by a late onset form that usually remains focal [6].

Available treatment options for ST include medical treatment (anticholinergic medications, levodopa, or the botulinum toxin type A), behavior modification (biofeedback, hypnosis, and exercises) and surgery [4].

Surgical approaches include non-specific and more specific therapies such as the DBS [3, 4].

DBS is an alternative and effective treatment in medication-resistant forms of dystonia [4]. It operates as a "brain pacemaker" and consists of three components: an intracranial insulated electrode implanted in the target neural tissue, a pulse generator-battery powered, positioned subcutaneously below the clavicle or in the abdomen, and an extension cable that connects the electrode to the battery [3].

As it concerns anesthesia, the characteristics of the disease and the respective treatment have to be considered.

Preoperative evaluation should focus on the severity of the dystonic movements. Airway management may be complicated because of prolonged abnormal posture of the head and in severe cases fiberoptic intubation may be indicated [6]. Mac et al concluded that patients with ST do not appear to have a higher frequency of difficult airway and intubation. A possible explanation is that the adverse influence of one or two factors (head rotation, limitation of extension) may be balanced by other favorable factors (adequate mouth opening). Muscle relaxants will also lead to better intubating conditions as the majority of patients will respond at least partially to these agents [7]. Our patient was intubated with the direct laryngoscopic technique in the first attempt and the intubation grade was Cormack I.

No anesthetic agents are contraindicated and non-depolarizing muscle relaxants abolish spasms and muscle rigidity that may remain after deactivation of the DBS. Propofol has been used successfully in the treatment of acute torticollis and $\mathrm{N}_{2} \mathrm{O}$ greater than $50 \%$ could improve the dystonic movements $[8,9]$. General endotracheal anesthesia in combination with muscle relaxants may be the preferred technique. Preoperative $\mathrm{X}$-ray examination traces the position of the leads and reveals the true nature of the implantable device. Precautions must be taken in order to avoid dysfunction of the generator and issue damage. DBS can interfere with electrocardiography, short wave diathermy, electrocautery, pacemakers and implantable defibrillators $[5,10]$.

DBS produces ECG artifacts and may make interpretation difficult. Deactivating the DBS system before ECG acquisition can remove such interference. This deactivation can cause severe tremor and electromyographic activity that could influence ECG recording and produce patient discomfort. The deactivation time may be short; however, patients may not be able to walk safely for the next hour [10]. Bipolar cautery is preferred because it reduces electromagnetic interference. Monopolar cautery must be used only in low voltage mode and with the lowest power settings. The ground plate should be placed as far from the pulse generator, the extension cable and the lead as possible. Diathermy energy could damage the brain at the site of implanted brain electrode as it might be 
transferred through the implanted system. Unlike pacemakers, these implants can be safely turned off for the duration of the operation $[5,10]$.

In our patient, diathermy plate was attached below his buttocks far from the DBS. We turned off the device right after induction, to minimize patient's discomfort from the muscle rigidity. After the completion of surgery, we turned on the device and with the evidence that the patient regained his muscle activity to facilitate smooth extubation and recovery. We antagonized the remaining neuromuscular blocking and our patient regained conscience and extubated $20 \mathrm{~min}$ after the activation of the DBS device without any discomfort.

In conclusion, our case report attempts to focus on the anesthetic implications of patients with a disease like ST that are treated with implantable devices like DBS. According to our experience, anesthetists should concentrate on a careful preanesthesia evaluation regarding primarily the airway examination, a detailed planning of the anesthetic technique to be applied, and a correct handling of the DBS implanted as it can interfere with other monitoring and therapeutic devices, sometimes with severe consequences.

\section{Conflicts of Interest}

None.

\section{References}

1. Zabani I, Vaghadia H. Refractory dystonia during propo- fol anaesthesia in a patient with torticollis-dystonia disorder. Can J Anaesth. 1996;43(10):1062-1064.

2. Fahn S, Bressman SB, Marsden CD. Classification of dystonia. Adv Neurol. 1998;78:1-10.

3. Venkatraghavan L, Luciano M, Manninen P. Review article: anesthetic management of patients undergoing deep brain stimulator insertion. Anesth Analg. 2010;110(4):1138-1145.

4. Smith DL, DeMario MC. Spasmodic torticollis: a case report and review of therapies. J Am Board Fam Pract. 1996;9(6):435-441.

5. Khetarpal M, Yadav M, Kulkarni D, Gopinath R. Anaesthetic management of a patient with deep brain stimulation implant for radical nephrectomy. Indian J Anaesth. 2014;58(4):461-463.

6. Scott BK, Baranov D. In: Fleisher LA, ed. Anesthesia and uncommon diseases. 6th ed. Elsevier-Saunders publishing, 2012:265-267.

7. Mac TB, Girard F, McKenty S, Chouinard P, Boudreault D, Ruel M, Bouvier G. A difficult airway is not more prevalent in patients suffering from spasmodic torticollis: a case series. Can J Anaesth. 2004;51(3):250-253.

8. Borgeat A. Usefulness of propofol in torticollis. $\mathrm{Br} \mathrm{J}$ Anaesth. 1991;66(4):530.

9. Martz DG, Schreibman DL, Matjasko MJ. Neurological diseases. In: Katz J, Benumof JL, Kadis LB (Eds.). Anesthesia and Uncommon Diseases. Philadelphia: W.B.Saunders Co, 1990:560-586.

10. Poon CC, Irwin MG. Anaesthesia for deep brain stimulation and in patients with implanted neurostimulator devices. Br J Anaesth. 2009;103(2):152-165. 